\title{
Impaired potentiation of H-reflexes in patients with upper motoneurone lesions
}

\author{
R. E. P. SICA, ${ }^{1}$ A. J. MCCOMAS ${ }^{2}$, AND A. R. M. UPTON ${ }^{2}$ \\ From the Regional Neurological Centre, Newcastle General Hospital, Newcastle upon Tyne, and the Nationals \\ Hospital for Nervous Diseases, Maida Vale, London
}

SUMMARY The potentiation of H-reflexes during effort has been studied in 16 patients with mild hemiparesis. Impaired potentiation was present in all but one patient, and was still evident in somee who had recovered fully from weakness. A tentative explanation has been proposed for these find- ings; it is suggested that there is loss of a background facilitatory pathway to motoneurones.

One of the cardinal features of a long-standing upper motoneurone lesion is that the stretch reflexes are exaggerated. In recent years it has been shown that this exaggeration is largely due to an increase in the sensitivity of the muscle spindle to passive stretch and that this, in turn, is a consequence of enhanced fusimotor ( $\gamma$-efferent) activity (Rushworth, 1960; see, however, Landau and Clare, 1964). Although the excitability of the fusimotor motoneurone is clearly raised, the situation in regard to the $\alpha$-motoneurone is more obscure. Until now the only method which has been used to investigate $\alpha$-motoneurone excitability in man has been that of $\mathrm{H}$-reflex testing. In this method the nerve to a muscle is stimulated electrically in a relaxed subject so that the $\alpha$-motoneurones receive monosynaptic excitation from Ia fibres; the ensuing reflex discharge is then monitored from the muscle. If measurement is also made of the maximal muscle response after direct excitation of $\alpha$-motor axons ( $M$ wave), then the fraction of motoneurones contributing to the recorded reflex potential will be H/M (Angel and Hofmann, 1963).

Unfortunately, for various reasons, the H-reflex method has not proved useful in the study of upper motoneurone disorders. In the first place, $\mathrm{H}$ reflexes cannot be detected at all in muscles other than those in the calf and sole, unless patients are quite severely affected. However, if the study is confined to calf muscles it suffers from the fact that even in healthy subjects the $\mathrm{H} / \mathrm{M}$ ratio may be unity (McComas and Payan, 1966). In other words, the entire motoneurone pool may be excited by an adequate

'Present address: Hospital Ramos Mejia, Buenos Aires, Argentina. 'Present address: Neurology Section, McMaster University Medical Centre, Hamilton, Ontario. stimulus, even without an upper motoneurone lesion. In addition Matthews (1966) has shown that intra- $-\oplus$ venous injection of diazepam or chlorproethazine may abolish spasticity without altering the $\mathrm{H} / \mathrm{H} \sim \overrightarrow{\mathrm{N}}$ ratio. An alternative approach has been to measu原 the recovery of excitability after an $\mathrm{H}$-refles. Although the recovery process is apparently somewhat faster in patients with upper motoneuron $\mathbb{D}$ lesions, the time course is complex and the method $\frac{\Phi}{3}$ is time-consuming.

We now wish to report the results of a new tye of study in such patients. This method also involves measurement of the H-reflex, but the results depend on the amount of background facilitation which can be imposed on $\alpha$-motoneurones during voluntary contraction. The method and the neural mechanisms underlying the evoked responses have been dealt? with in the accompanying paper (Upton, McComas, $\frac{0}{\mathbb{Q}}$ and Sica, 1971); a preliminary account has already appeared (McComas, Sica, and Upton, 1970).

It will be shown that in most hemiparetic patients there is a characteristic inability to potentiate $\mathrm{H}$ reflexes in an affected limb. Furthermore, this impairment is still present in patients in whom an apparently complete recovery from weakness haso occurred. The findings have been sufficiently striking to suggest that the method may find a useful place in the diagnosis of neurological disorders.

\section{METHODS}

PATIENTS Investigations were made in 11 women and six음 men with evidence of upper motoneurone disturbance affecting one or both limbs on one side only; their ages $\mathrm{N}$ ranged from 20 to 66 years. Five of these patients had suffered from transient attacks of cerebral ischaemia; i: 
the remaining patients the diagnoses included glioma (2), meningioma (1), cerebral thrombosis (5), cerebral embolism (2), and subarachnoid haemorrhage (2). In each patient a clinical assessment was made of the tendon jerks, limb tone, and the plantar reflex. Muscle strength was judged clinically and, in most cases, with a springbalance. In eight patients in whom there had been a definite history of transient limb weakness due to cerebrovascular disease, the physical findings in the affected limbs were entirely normal at the time of investigation. Both arms were also normal in another patient in whom a cerebral glioma was causing slight weakness of one leg. The experimental results in this group of nine patients are shown separately in Fig. 2. In the remaining patients only mild weakness was demonstrable. Control observations were made in 23 healthy adults, aged 19 to 49 , who were subjects in the initial study of late wave potentiation (Upton et al., 1971).

EXPERIMENTAL PROCEDURE Late wave potentiation was studied in corresponding muscles of the affected and normal limbs. In 14 patients the abductor pollicis brevis (APB) was used; four further experiments were performed on the tibialis anterior (TA) or the abductor digiti minimi (ADM). The techniques for stimulating and recording from these muscles have already been described (Upton et al., 1971). In the present experiments the peak-to-peak amplitudes of $10 \mathrm{~F}$ waves were measured with the subject relaxed. The subject then performed a maximal contraction of the muscle under examination and the amplitudes of $10 \mathrm{~V}_{1}$ (potentiated $\mathrm{H}$-reflex) waves were recorded. The stimuli to the appropriate mixed nerve were kept supramaximal for both series of observations and the repetition 1 ate was less than one every three seconds.

\section{RESULTS}

In a normal subject at rest, supramaximal nerve stimulation usually evokes a small $\mathrm{F}$ wave in a distal

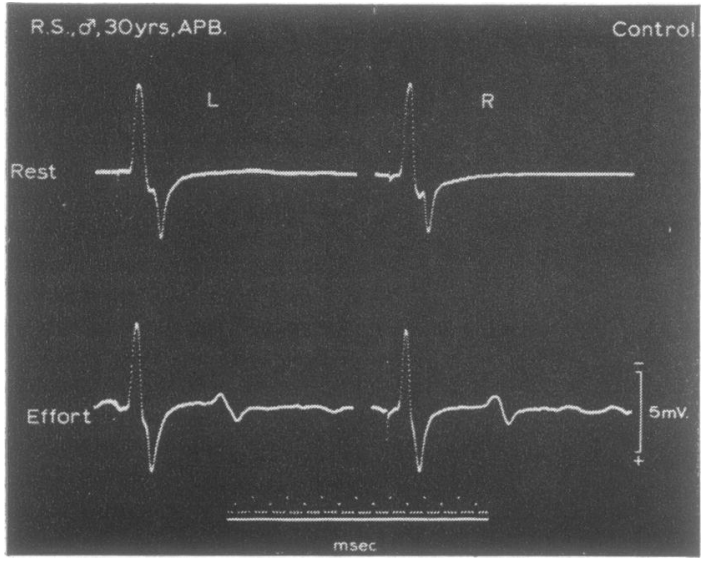

FIG. 1A muscle. When the subject contracts the muscle voluntarily a large evoked response appears which has a similar latency to that of the $F$ wave. In the accompanying paper (Upton et al., 1971) this latter response was designated the $V_{1}$ (first volitional) wave and further analysis showed that it consisted mainly of a potentiated $\mathrm{H}$-reflex. In most normal subjects a second wave can also be detected on effort. This second $\left(\mathrm{V}_{2}\right)$ wave will not be considered further in the present paper, although it was apparent that the

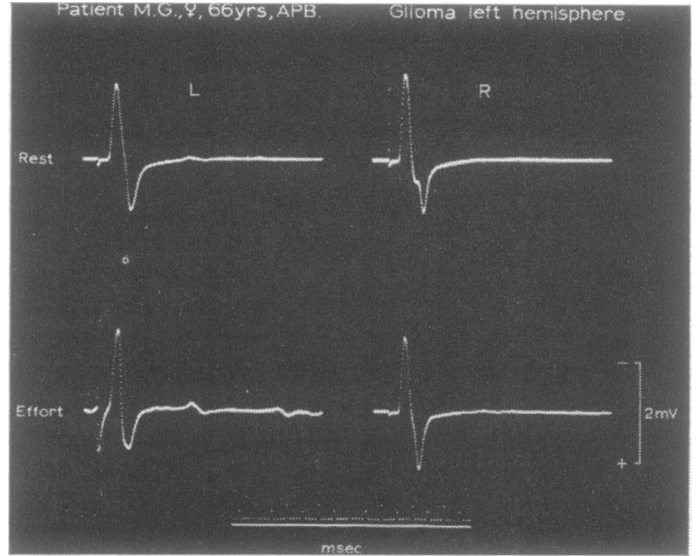

FIG. 1B

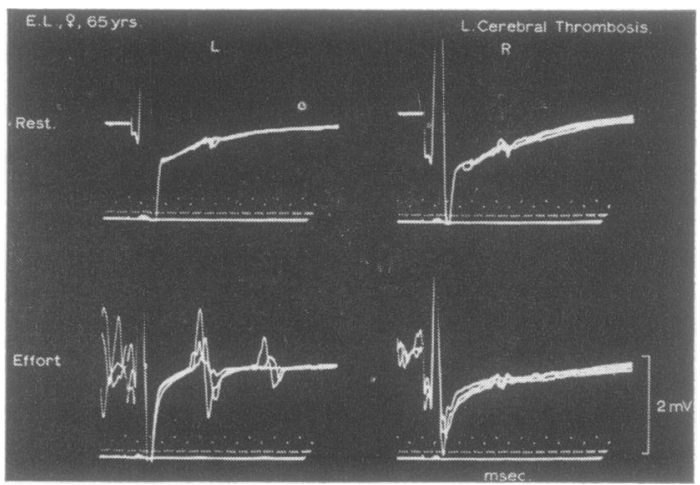

FIG. $1 \mathrm{C}$

FIG. 1. Potentiation of 'late' responses compared in the two APB muscles of a control subject (Fig. 1A) and two patients with upper motoneurone lesions (Figs. 1B, C). In each subject the effort was maximal. In $\mathrm{A}$ and $\mathrm{B}$ each trace shows the average of 10 responses; in $\mathrm{C}$ several consecutive traces have been superimposed. When measurements of $V_{1}$ responses were made at higher gain the symmetry coefficients of these subjects were 98 (A), 10 (B), and $-24(\mathrm{C})$. 
neural mechanisms responsible for this wave could be affected by upper motoneurone lesions-for example, Fig. 1c.

In the case of the first wave, the extent of potentiation in any one muscle will be the difference between the $V_{1}$ wave during effort and the $F$ wave at rest. This difference may be expressed in terms of the maximum $M$ wave, measured at rest, so as to give an excitability coefficient, $Z$,

$$
\text { where } Z=\frac{V_{1}-F}{M}
$$

Using the excitability coefficients it is possible to compare the abilities of corresponding muscles on both sides to potentiate. Thus, if the smaller excitability coefficient $(Z)$ is expressed as a percentage of the larger $\left(\mathbf{Z}^{\prime}\right)$, a symmetry coefficient $(\mathbf{S})$ is obtained,

$$
\text { that is, } S=\frac{Z}{Z^{\prime}} \times 100 \text {, where } Z<Z^{\prime}
$$

In a healthy subject, provided the voluntary contraction is maximal, the average amount of potentiation is very similar on both sides of the body. An example of this symmetry is given in Fig. $1 \mathrm{~A}$ where $\mathrm{F}$ waves and $V_{1}$ waves have been averaged automatically in each abductor pollicis brevis (APB) muscle of the control subject R.S. Individual values for the symmetry coefficients in this and other control subjects have been arranged in ranking order in Fig. 2. Additional observations have been made in the first dorsal interosseous muscle (DI) of 11 subjects and in the extensor digitorum brevis (EDB) muscles of six subjects. In DI the symmetry coefficients ranged from 82 to 98 with a mean of $91 \mathrm{SD} \pm 5 \cdot 1$. क In EDB the range was 70 to 96 and the mean wasō $87 \pm 9 \cdot 8$.

In the patients with upper motoneurone lesions $x \overline{\overline{0}}$ different picture emerged. In relaxed muscles the ${ }_{\mathcal{D}}$ $F$ waves on the affected side were usually of normal size; however, in one patient-a 65 year old man with a cerebral glioma, an abnormally large potential was recorded $(\mathrm{F} / \mathrm{M}=26 \%$; see Fig. 3). Although: the stimulus was supramaximal it was nevertheless possible that a large part of this enhanced potentiato was an H-reflex. Some motoneurones may have dis-흠 charged reflex impulses too late for collision to have $\frac{\bar{p}}{\bar{D}}$ occurred with the antidromic volley (see also Upton $\mathbb{\odot}$ et al., 1971). When the patients made maximalo voluntary contractions the $\mathrm{V}_{1}$ waves were usuallys very much larger on the normal side than on the. affected one, even in patients with no demonstrable $\vec{\overrightarrow{ }}$ abnormality in the previously weak limb. Two. examples of unequal potentiation are given in Fig. 1. Although this disparity between the two sides is 0 evident from Fig. 3, it is shown most clearly in Fig. $2 \dot{\omega}$ where the symmetry coefficient has been calculatedos for each patient and compared with the values ip $-\mathrm{J}$ controls. Only one patient had a coefficient $(86) \sim$ above the lower limit of the normal range $(6 \%$.을 This patient was a 63 year old man with a cerebralglioma. It can also be seen from Fig. 2 that the symmetry coefficient was occasionally negative, since in some patients the late response actually declined 3 effort. The cause of this diminution was uncerta It may have been due to refractoriness of motor $\vec{c}$ axons after 'volitional' impulses or it may have
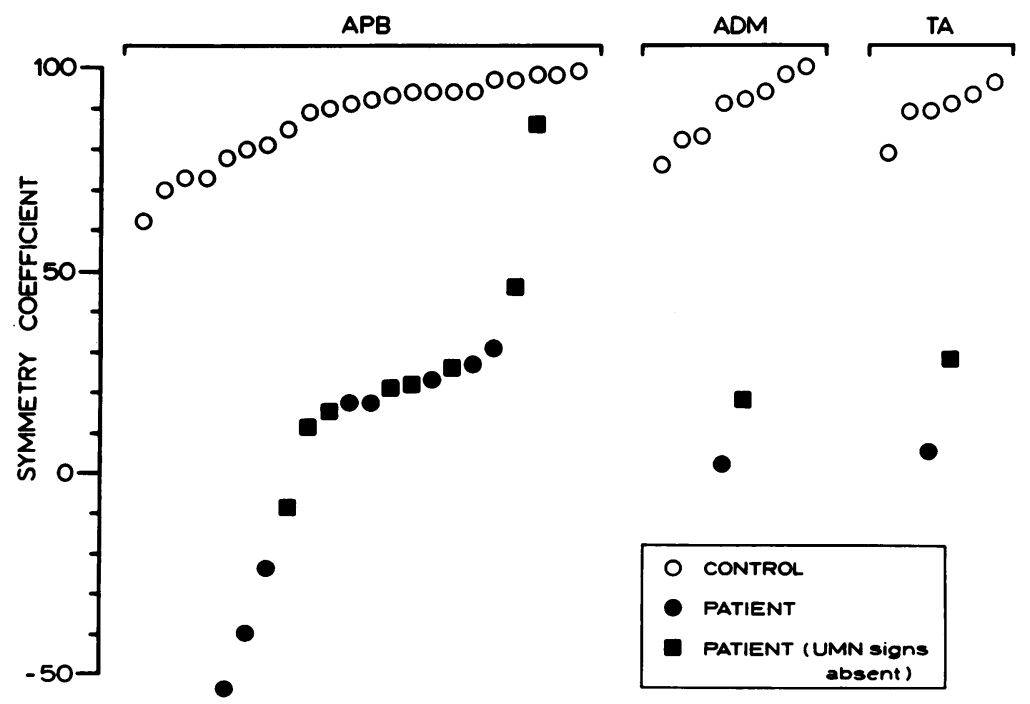

FIG. 2. Symmetry coefficients arranged in ranking order for $\frac{7}{0}$ APB, ADM, and TA muscles of control subjects (open circles), patients with mild weakness (filled circles), and patients with no detectable abnormality in the investigated limbs (filled squares). See text. 

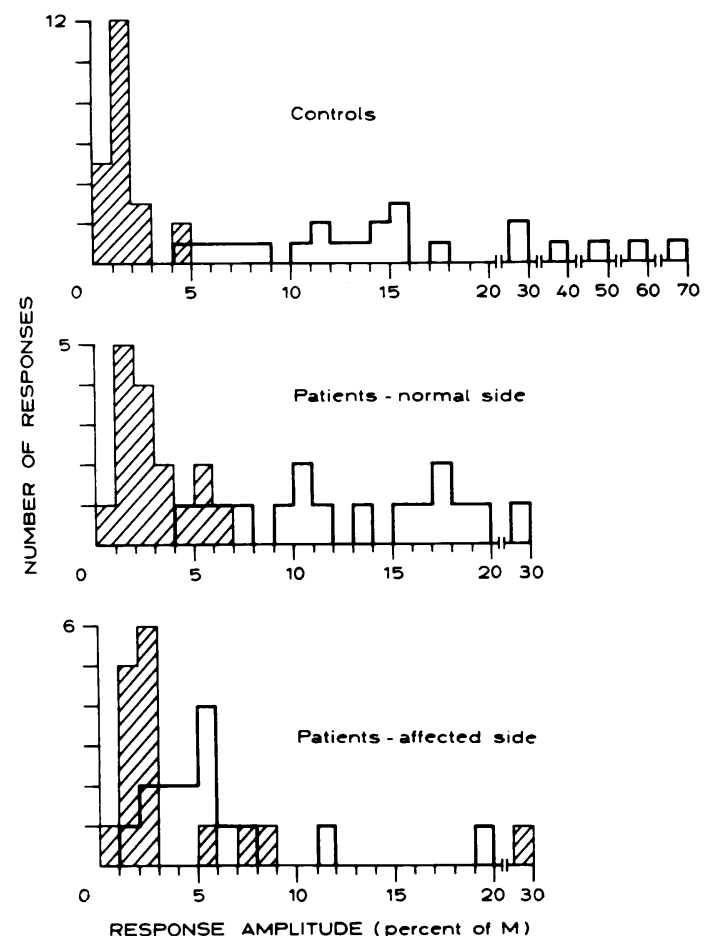

$\square$ F wave $\square$ vi wave

FIG. 3. Amplitudes of $F$ waves during rest (hatched columns) and of $V_{1}$ waves during maximum effort (open columns) in APB muscles of patients and controls.

reflected the operation of inhibitory processes during effort.

\section{DISCUSSION}

In the introduction attention was drawn to the difficulties inherent in conventional $\mathrm{H}$-reflex investigations of patients with upper motoneurone lesions. A more promising approach has been to see if the H-reflex potentiated in paretic muscles during the Jendrassik manoeuvre. Even in this type of study the results have been conflicting. Thus, whereas Buller (1957) found that reinforcement was diminished or absent, Landau and Clare (1964) showed that the H-reflex could be enhanced provided the stimulus was submaximal. Our previous experience with the Jendrassik manoeuvre yielded inconsistent results, since in normal subjects the manoeuvre sometimes caused a reduction in the size of the $\mathrm{H}$ reflex (McComas and Payan, 1966). Even in the same subject the effect of the manoeuvre might vary on different occasions. In view of this uncertainty in normal subjects, the Jendrassik manoeuvre is unlikely to form a useful test for the integrity of descending pathways.

The present technique differs from the others considered in so far as it embodies voluntary muscular contraction as a facilitatory mechanism for the H-reflex. The results obtained suggest that the new method forms an extremely sensitive test of upper motoneurone function. We have found that, in all but one of 17 patients with mild upper motoneurone lesions, there was a significant failure to potentiate the $\mathrm{H}$-reflex on the affected side. The most striking observation was that eight of the patients, all with impaired potentiation, were judged to have recovered completely from a previous episode of weakness.

In the execution of our test we have preferred to use supramaximal, rather than submaximal, nerve stimulation for two reasons. First, the movements of synergistic muscles and their tendons during a volitional contraction inevitably alter the positions of the stimulating electrodes in relation to the nerve. Hence, a constant submaximal stimulus cannot be maintained. Secondly, if submaximal stimulation is used, the volitional activity in the recorded electromyogram makes the measurement of evoked potentials difficult. One solution is automatic averaging of the electromyogram but, even with this, background activity may still be apparent in the averaged record (cf. Fig. 4 of Upton et al., 1971). On the other hand, although the H-reflex is markedly attenuated by a supramaximal stimulus, the antidromic volley temporarily prevents 'volitional' impulses from reaching the muscle; consequently the evoked potentials are super-imposed on a flat record-for example, Fig. 1. Furthermore, even if the electrodes move during the contraction, a sufficiently large stimulus will still remain supramaximal for motor axons.

Finally, it remains to consider the nature of the upper motoneurone disturbance which is responsible for the impaired potentiation. At first sight the findings are paradoxical since motoneurone excitability, assessed in terms of volitional activity, may be considered normal if there is no impairment of strength in the investigated muscle. When Ia inputs are used as the testing procedure in such a muscle, however, motoneurone excitability is usually depressed.

There are at least two ways in which an apparently conflicting situation might arise. The first possibility depends on the observation that the efficacy of the Ia endings are normally under the control of presynaptic inhibitory mechanisms, which themselves receive inputs from higher centres (Carpenter, 

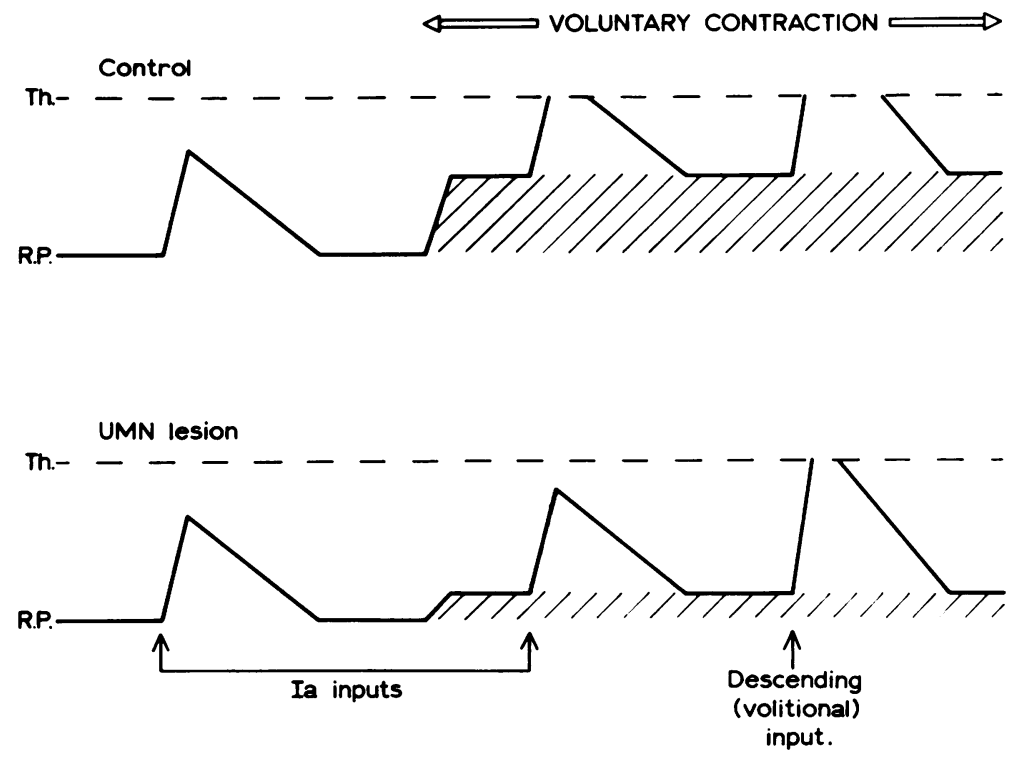

FIG. 4. Postulated potentiating mechanism. Ordinate is membrane potential of a motoneurone in control subject (upper) and patient (lower); interrupted line denotes threshold (Th) for initiation of action potentials while R.P. indicates resting potential. Time is represented by abscissa. During voluntary contraction (arrow's at top) the motoneurone is subjected to a background depolarization (hatched area) which is smaller in the patient than in the control.

Engberg, and Lundberg, 1966). If, during contraction in a healthy subject, the presynaptic mechanisms are held in abeyance by descending pathways then this restraining influence-that is, 'disinhibition'-might be absent after an upper motoneurone lesion. Consequently, electrically-induced Ia fibre inputs would be less effective in patients than in controls. Stretch reflexes would still be exaggerated, however, due to the overriding influence of the increased fusimotor activity. Provided that sufficient descending channels remained intact after the lesion, volitional activity would be unaffected. A similar explanation could be based on temporary suppression of postsynaptic inhibitory neurones, such as those innervated by Ib (Golgi tendon organ) fibres.

The second possibility is rather simpler and has therefore been tentatively accepted as our working hypothesis; it is summarized diagrammatically in Fig. 4. It supposes that during a voluntary contraction one group of descending fibres normally evokes excitatory postsynaptic potentials (EPSPs) repetitively so as to produce a background, but subthreshold, depolarization of the motoneurone. Superimposed on this background depolarization are relatively large EPSPs from other descending fibres which cause the cell to fire action potentials.
In the presence of this background depolarization. Ia inputs may alsoelicitimpulses in the motoneurone In upper motoneurone lesions of the type exhibited by our patients, we postulate that the subthreshold depolarization is reduced. Even though Ia inputs will no longer evoke reflex discharges, the volitional pathways remain effective because of the high safety margin of the corresponding EPSPs.

If the last mechanism is the correct explanation of our findings, it remains to identify the descending pathway(s) normally responsible for the subthreshold effects. In the accompanying paper (Upton et al., 1971) we have shown that the descending pathway does not depend on the stretch reflex loop. In an attempt to obtain further information we have recently undertaken a study in patients with Parkinsonism, some of whom have been treated by thalamotomy. The results of these further investigations will follow in due course (McComas, Upton, Hankinson, Petito, and Hughes, 1971).

We are grateful to Professor J. N. Walton, Dr. J. B. Foster, and Dr. D. D. Barwick for permission to study $N$ their patients. In addition, we wish to thank Mr. T. Blogg os and Mr. C. Wallace for technical services and Mrs. Y. N Chisholm for secretarial assistance. Financial support N 
was received from the Muscular Dystrophy Group of Great Britain, the Muscular Dystrophy Associations of America Inc., and the Medical Research Council. One of us (R.E.P.S.) held a British Council Scholarship.

\section{REFERENCES}

Angel, R. W., and Hofmann, W. W. (1963). The H-reflex in normal, spastic, and rigid subjects. Arch. Neurol. (Chic.), 8, 591-596.

Buller, A. J. (1957). The ankle-jerk in early hemiplegia. Lancet, 2, 1262-1263.

Carpenter, D., Engberg, I., and Lundberg, A. (1966). Primary afferent depolarization evoked from the brain stem and the cerebellum. Arch. ital. Biol., 104, 73-85.

Landau, W. M., and Clare, M. H. (1964). Fusimotor function. Part VI. Reflex, tendon jerk, and reinforcement in hemiplegia. Arch. Neurol. (Chic.), 10, 128-134.
Matthews, W. B. (1966). Ratio of maximum H reflex to maximum $\mathbf{M}$ response as a measure of spasticity.J. Neurol. Neurosurg. Psychiat., 29, 201-204.

McComas, A. J., and Payan, J. (1966). Motoneurone excitability in the Holmes-Adie syndrome. In Control and Innervation of Skeletal Muscle. A Symposium, pp. 182193, edited by B. L. Andrew. Livingstone: Edinburgh.

McComas, A. J., Sica, R. E. P., and Upton, A. R. M. (1970). Excitability of human motoneurones during effort. $J$. Physiol. (Lond.), 210, 145-146P.

McComas, A. J., Upton, A. R. M., Hankinson, J., Petito, F., and Hughes, R. C. (1971). Potentiation of 'late' muscle responses in Parkinsonism. In preparation.

Rushworth, G. (1960). Spasticity and rigidity: an experimental study and review. J. Neurol. Neurosurg. Psychiat., 23, 99-118.

Upton, A. R. M., McComas, A. J., and Sica, R. E. P. (1971). Potentiation of 'late' responses evoked in muscles during effort. J. Neurol. Neurosurg. Psychiat., 34, 699-711. 